Supporting Information Section

\title{
A Facile Method for the Synthesis of Thiocarbamates: Palladium- Catalyzed Reaction of Disulfide, Amine and Carbon Monoxide
}

\author{
Yutaka Nishiyama*, Hiroaki Kawamatsu and Noboru Sonoda*
}

Department of Applied Chemistry, Faculty of Engineering, Kansai University, Suita, Osaka 5648680 , Japan

nishiya@ipcku.kansai-u.ac.jp

Table of Contents

Experimental Methods

$S$-t-Butyl- $N, N$-diethylthiocarbamate (3j)

$S$-Cyclohexyl- $N, N$-diethylthiocarbamate (3k)

References

Spectra for $S$-4-Methoxyphenyl- $N, N$-diethylthiocarbamate (3e)

Spectra for $S$-s-Butyl- $N, N$-diethylthiocarbamate (3i)

Spectra for $S$ - $t$-Butyl- $N, N$-diethylthiocarbamate (3j)

Spectra for $S$-Cyclohexyl- $N, N$-diethylthiocarbamate (3k)

Spectra for S-Phenyl-N,N-ethyl-i-propylthiocarbamate (3m)
S1

S1

S1

S2

S3-S5

S6-S8

S9-S11

S12-S14

S15-S17

General procedure:

${ }_{1} \mathrm{H}-$ and ${ }_{13} \mathrm{C}-\mathrm{NMR}$ spectra were recorded on 400 and $270 \mathrm{MHz}$ spectrometers using $\mathrm{CDCl}_{3}$ as solvent with tetramethylsilane as the internal standard. IR spectra were obtained on an FT-IR spectrophotometer. Mass spectra were measured by GC-MS. Gas chromatography $(\mathrm{GC})$ was carried out on an instrument with a flame-ionizing detector using a capillary column. Diaryl and dialkyl disulfides, and palladium complexes were commercially available and used without purification. Amines and solvents were purified by the usual methods.

$S$ - $t$-Butyl- $N, N$-diethylthiocarbamate $(3 \mathrm{j})$ ${ }_{1} \mathrm{H}-\mathrm{NMR}\left(\mathrm{CDCl}_{3}\right)_{-} 1.15(\mathrm{t}, \mathrm{J}=6.8 \mathrm{~Hz}, 6 \mathrm{H}), 1.51(\mathrm{~s}, 9 \mathrm{H}), 3.48(\mathrm{q}, \mathrm{J}=6.8 \mathrm{~Hz}, 4 \mathrm{H}) .{ }_{13 \mathrm{C}}$ NMR (CDCl 3 )_13.5, 30.6, 47.2, 167.2. IR (neat) 859, 1112, 1248, 1361, 1403, 1460, $1644,1742,2854,2925 \mathrm{~cm}-1$. Anal. Calcd for $\mathrm{C}_{9 H}{ }_{19} \mathrm{NOS}$ : C, 57.10; H, 10.12; N, 7.40. Found: C, 57.26; H, 10.45; N, 7.26.

\section{$S$-Cyclohexyl- $N, N$-diethylthiocarbamate (3k)}

${ }_{1} \mathrm{H}-\mathrm{NMR}\left(\mathrm{CDCl}_{3}\right)-1.16(\mathrm{t}, \mathrm{J}=6.4 \mathrm{~Hz}, 6 \mathrm{H}), 1.12-1.39(\mathrm{~m}, 1 \mathrm{H}), 1.43(\mathrm{q}, \mathrm{J}=6.4 \mathrm{~Hz}, 4 \mathrm{H})$, $1.57-1.61(\mathrm{~m}, 1 \mathrm{H}), 1.69-1.77(\mathrm{~m}, 2 \mathrm{H}), 1.98-2.02(\mathrm{~m}, 2 \mathrm{H}), 3.36-3.45(\mathrm{~m}, 5 \mathrm{H}) .13 \mathrm{C}-\mathrm{NMR}$ (CDCl 3 ) 13.4, 25.7, 26.2, 33.9, 42.1, 43.6, 166.9. IR (neat) 665, 755, 859, 1097, 1116, $1221,1248,1380,1405,1449,1650,2853,2932,2974 \mathrm{~cm}-1$. Anal. Calcd for $\mathrm{C}_{11} \mathrm{H}_{21} \mathrm{NOS}$ : C, 61.35; H, 9.83; N, 6.50. Found: C, 61.74; H, 9.65; N, 6.71. 


\section{References}

1) Mizuno, T.; Nishiguchi, I.; Hirashima, T. Tetrahedron 1993, 49, 2403.

2) Beaulieu, F.; Snieckus, V. Synthesis 1992, 112.

3) Minnemeyer, H. J.; Clarke, P. B.; Tieckelmann, H. J. Org. Chem. 1966, 31, 406.

4) Kuniyasu, H.; Hiraike, H.; Morita, M.; Tanaka, A.; Sugoh, K.; Kurosawa, H. J. Org. Chem. 1999, 64, 7305.

5) Miyazaki, K. Tetrahedron Lett. 1968, 2793.

6) Kim, Y.-H; Chung, B.-C; Chang, H.-S. Tetrahedron Lett. 1985, 26, 1079. 


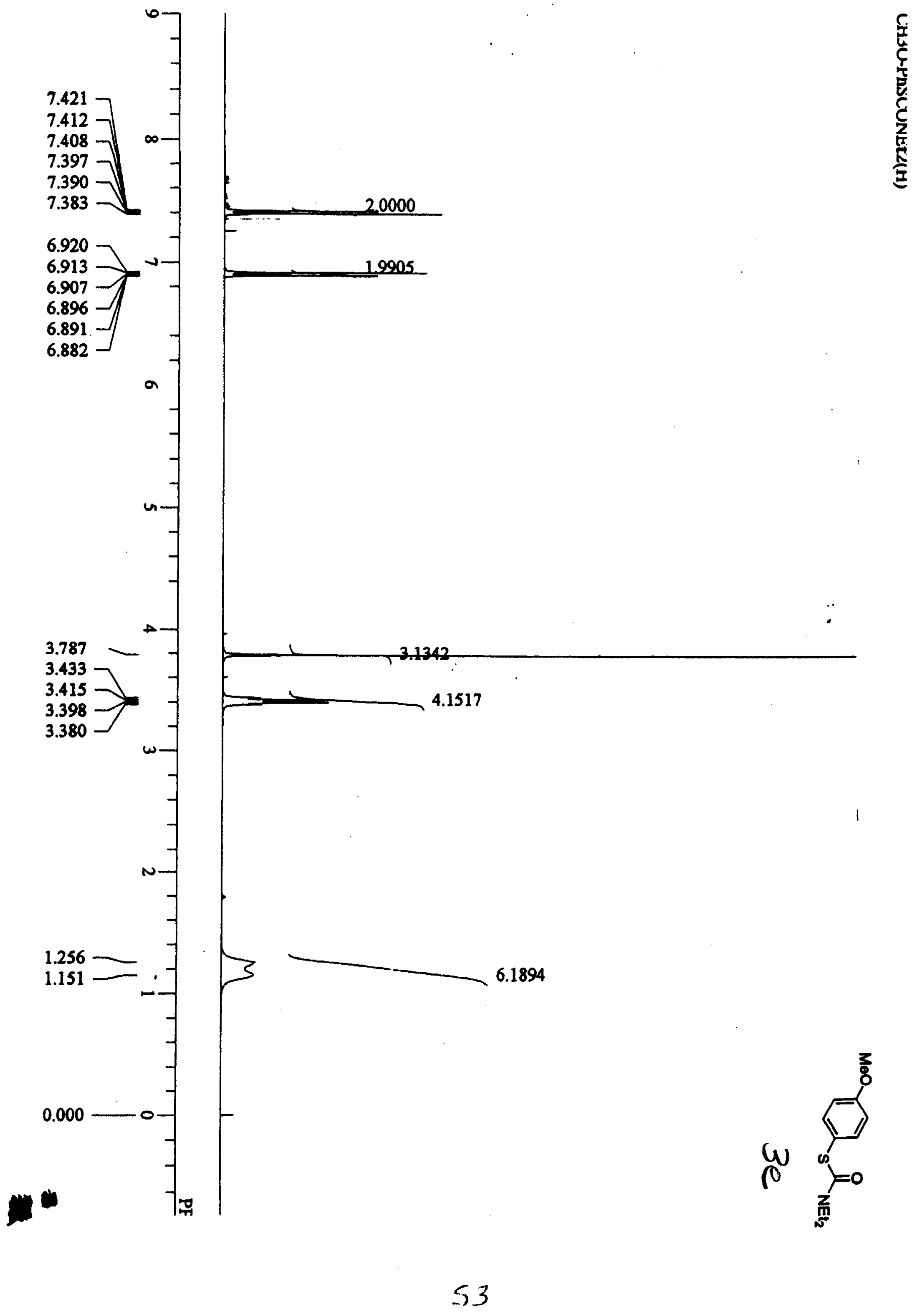




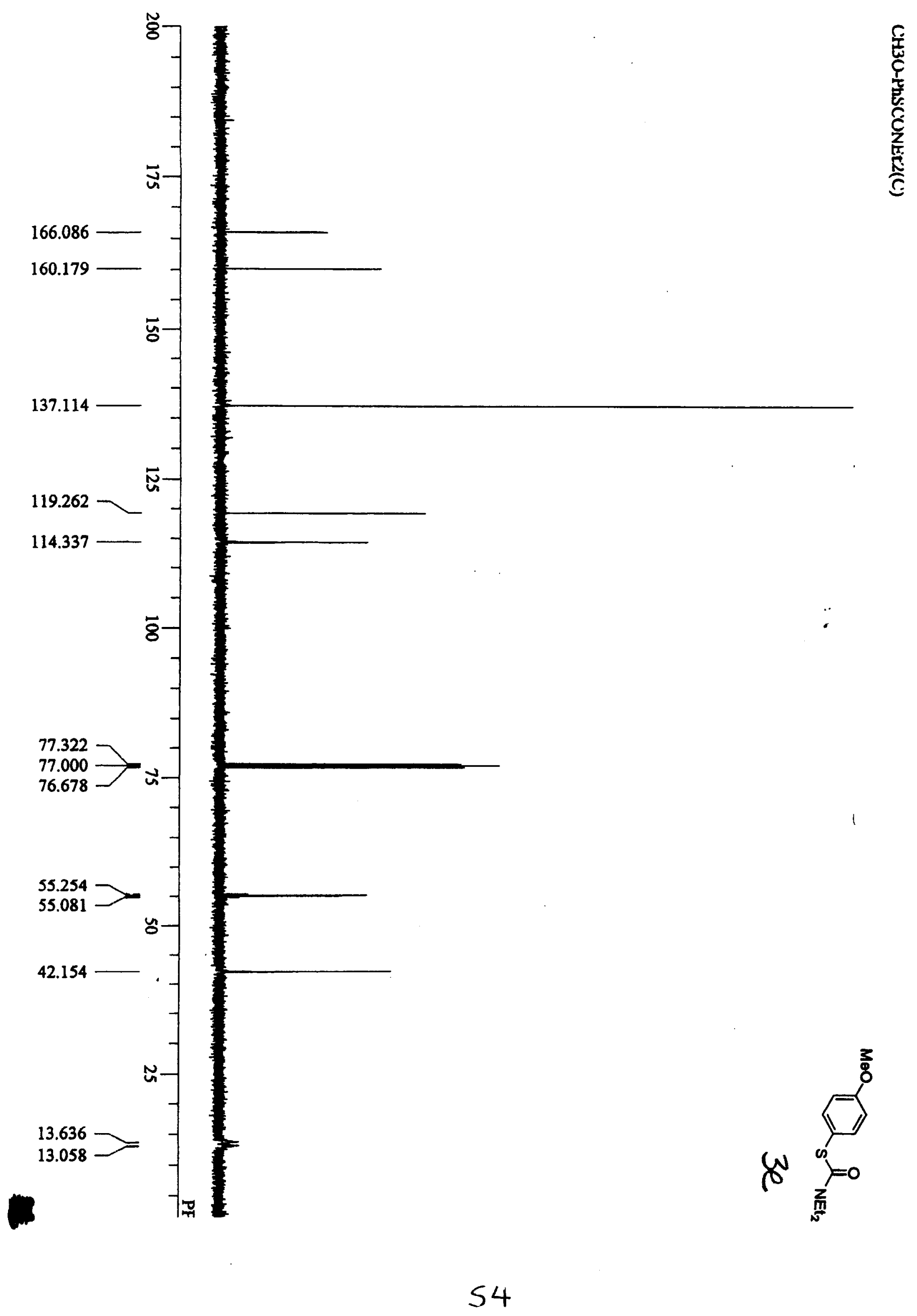




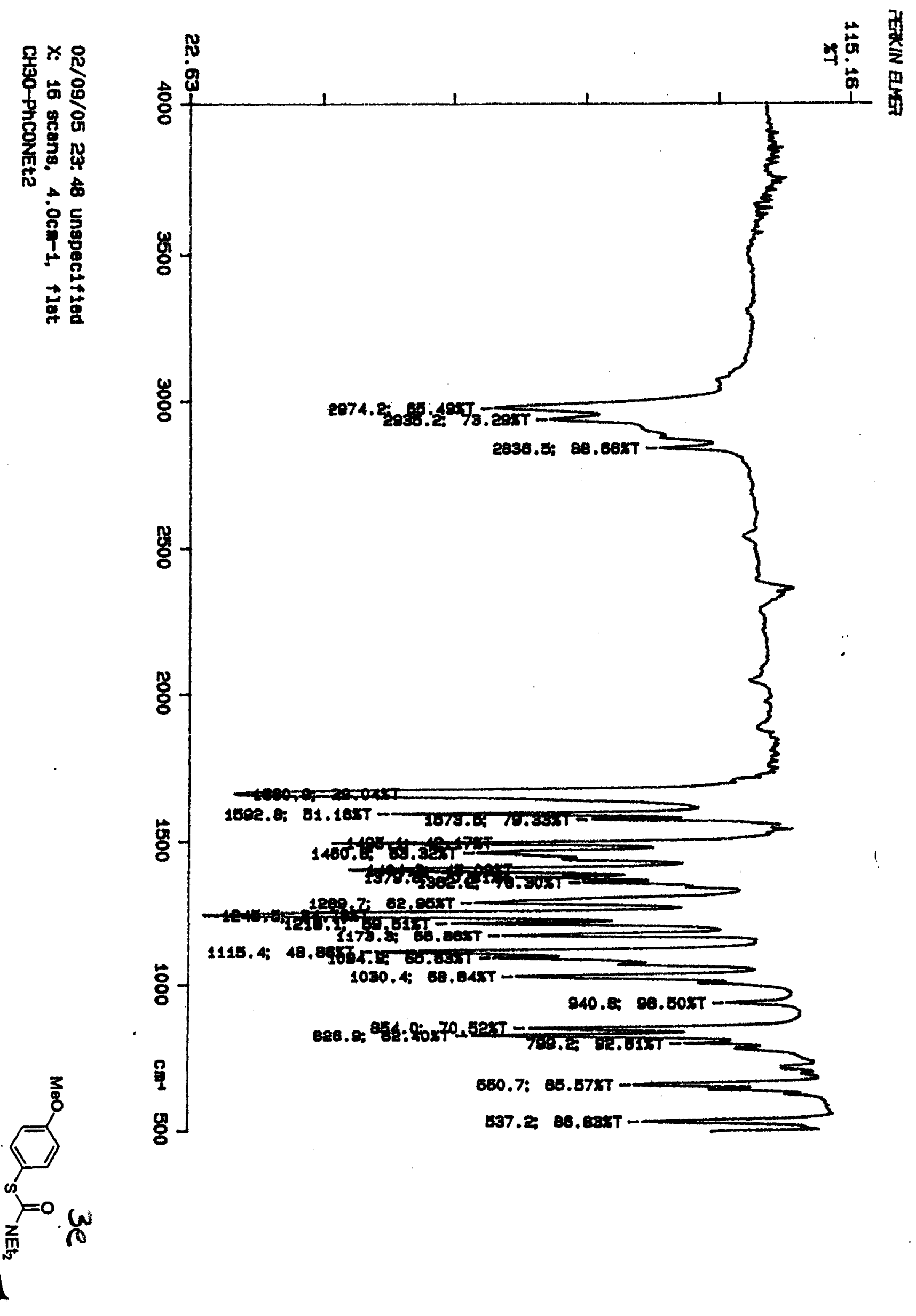




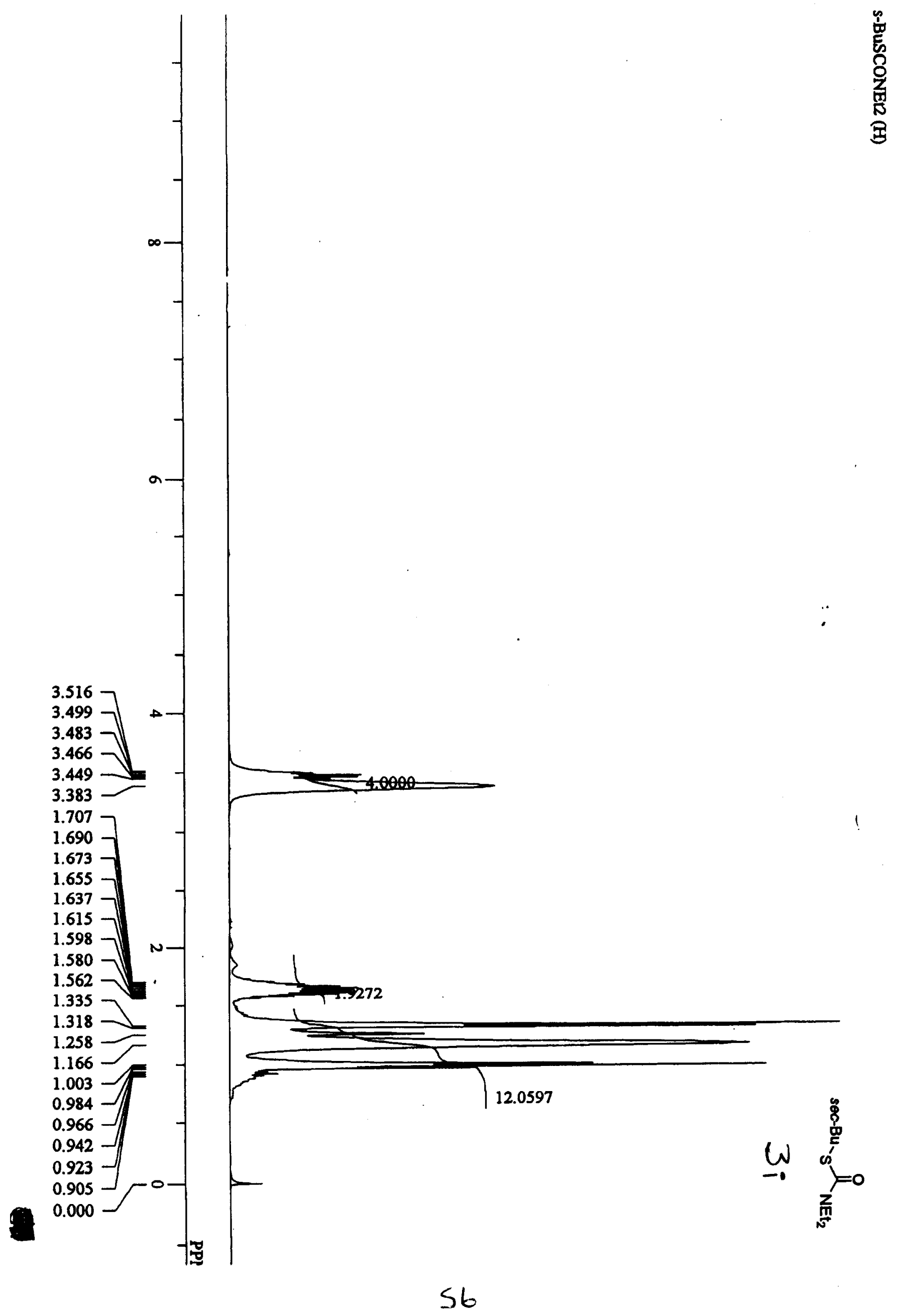




$$
=
$$




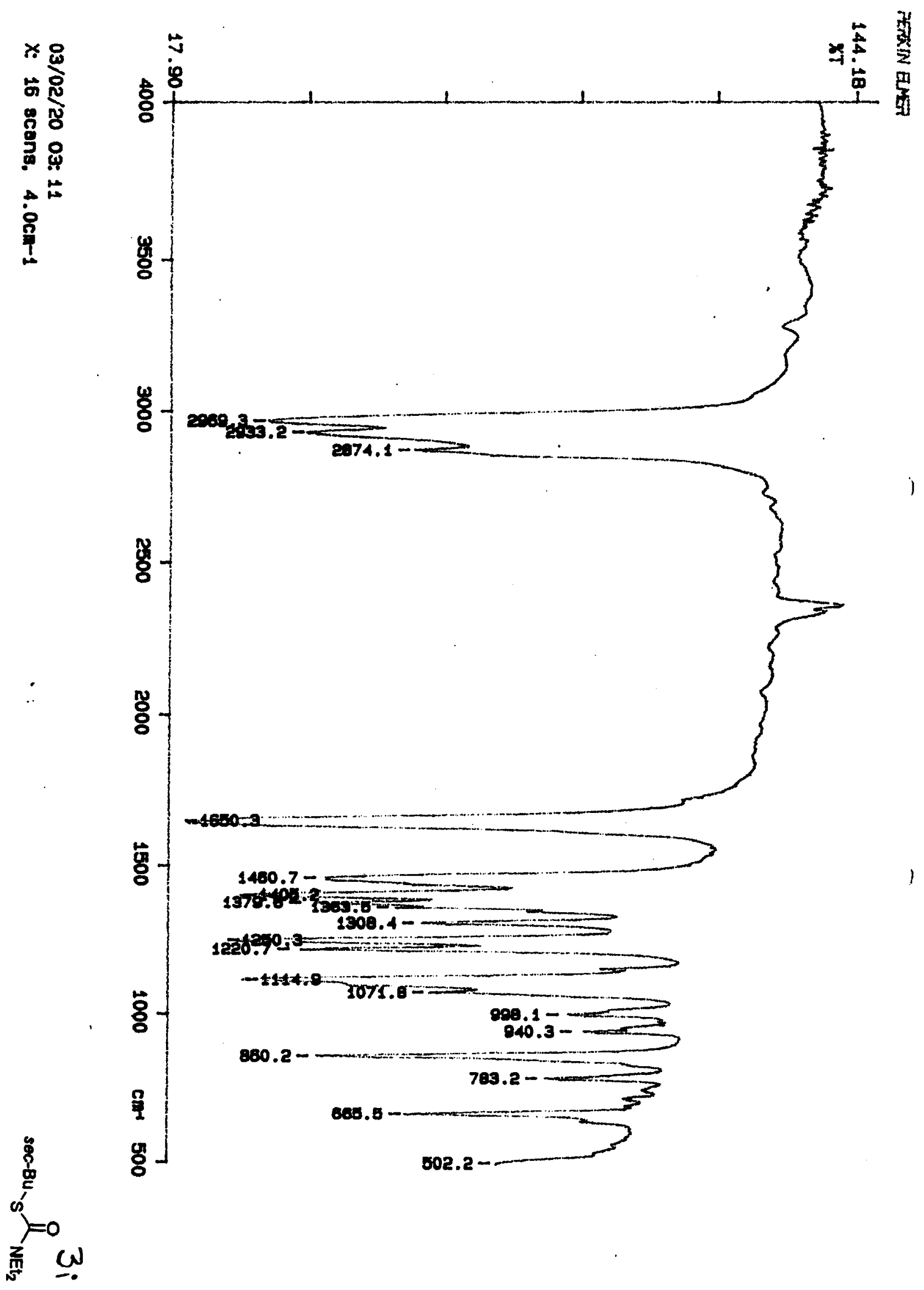

58 


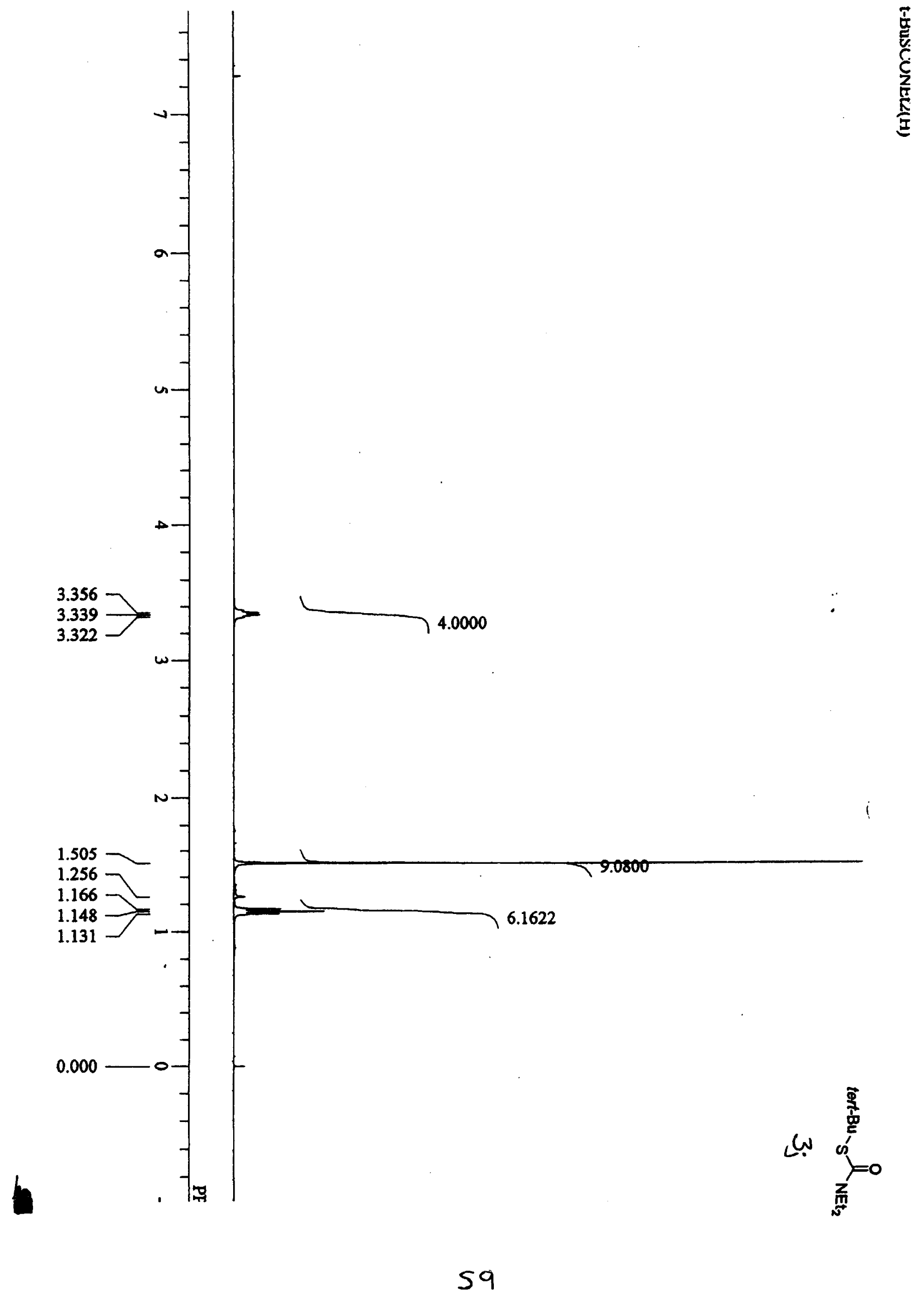




$$
1
$$




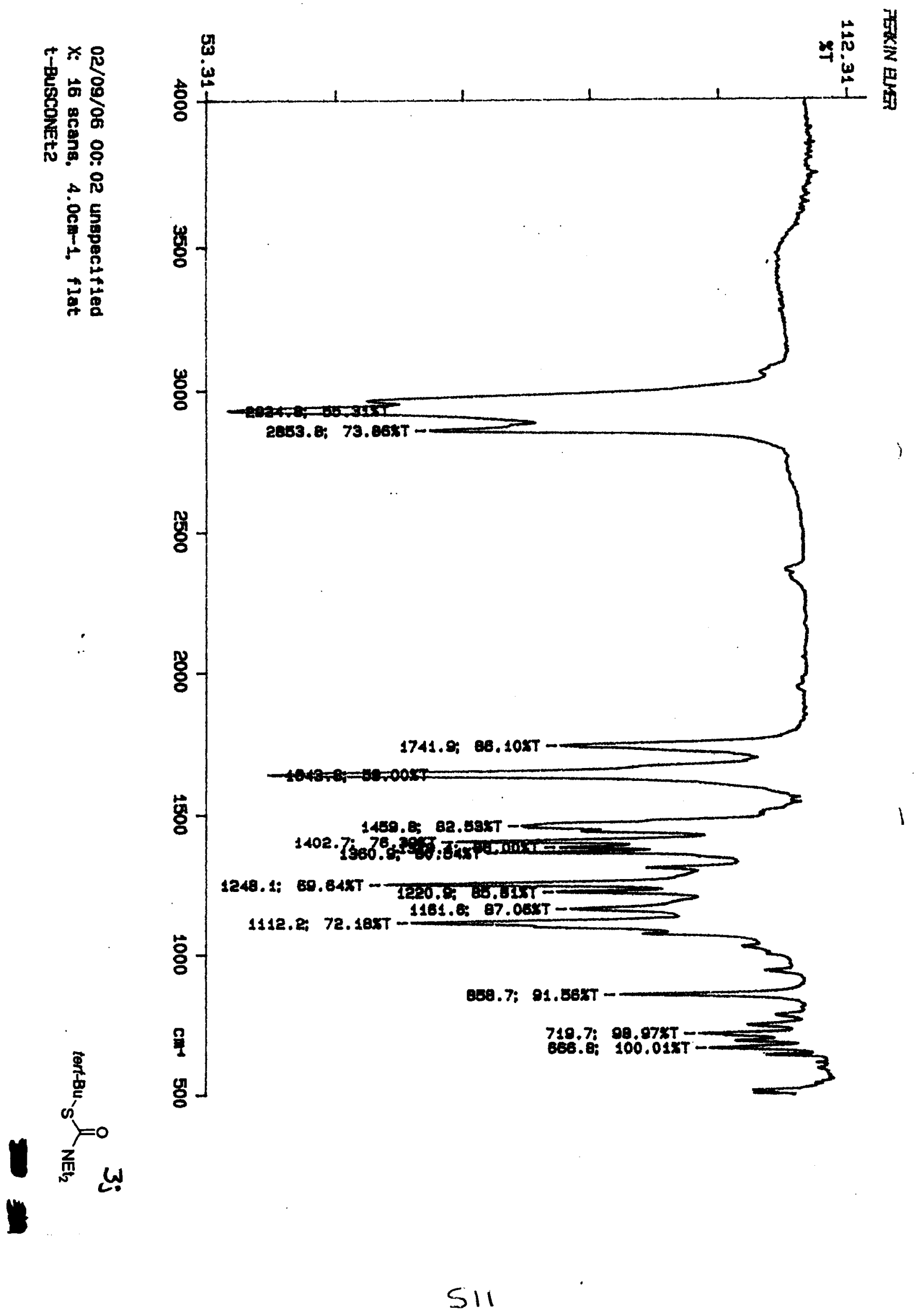




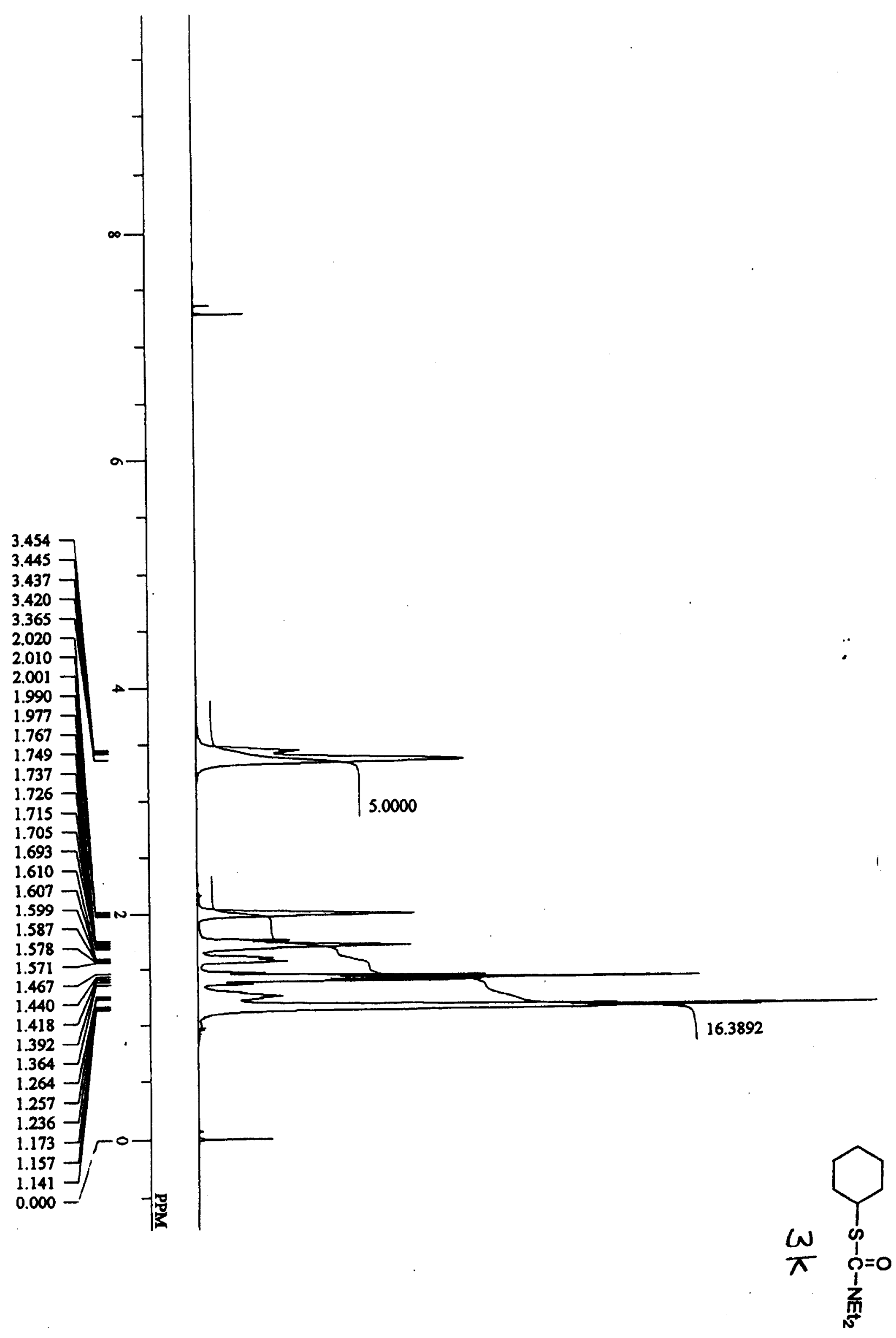

512 


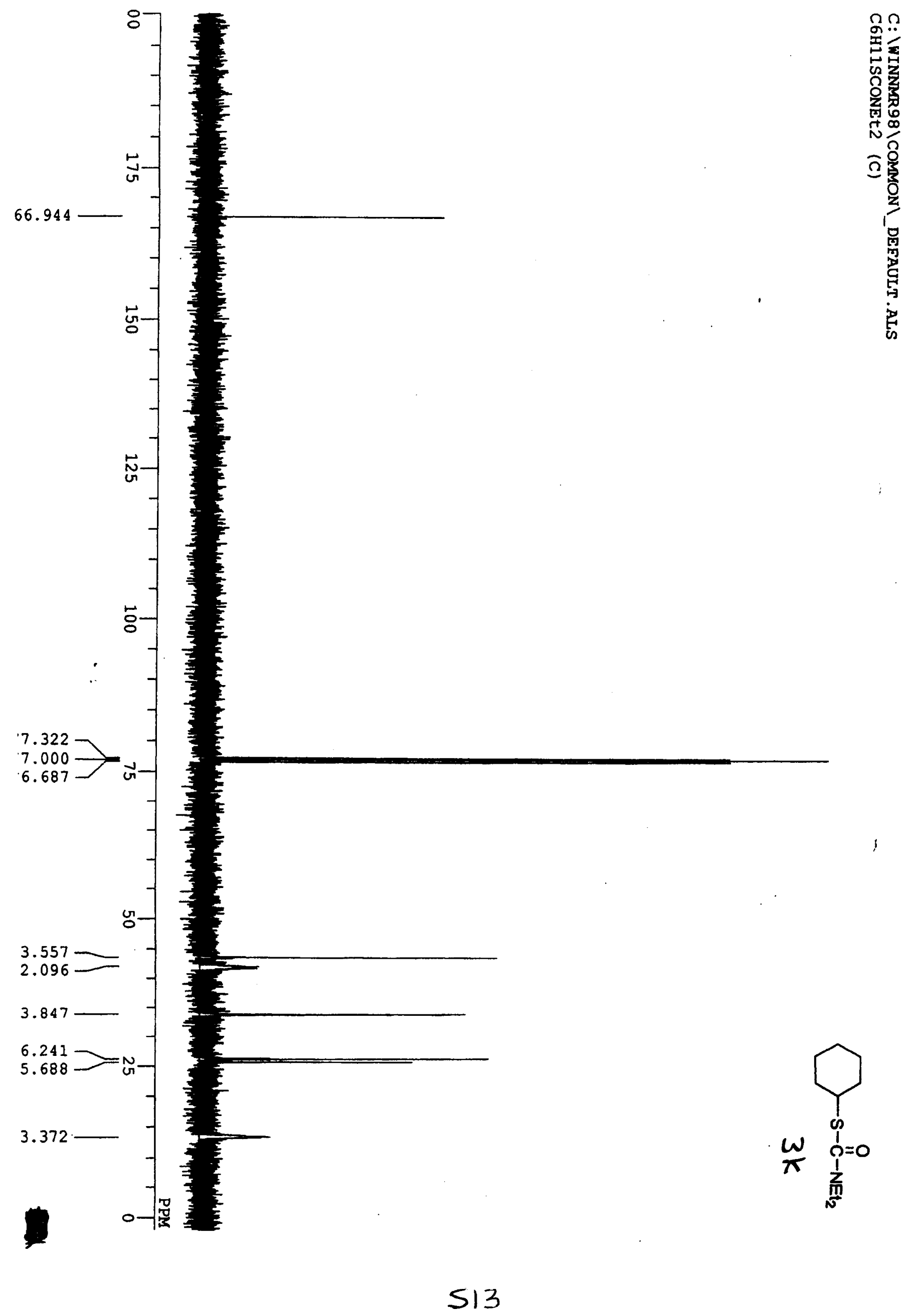




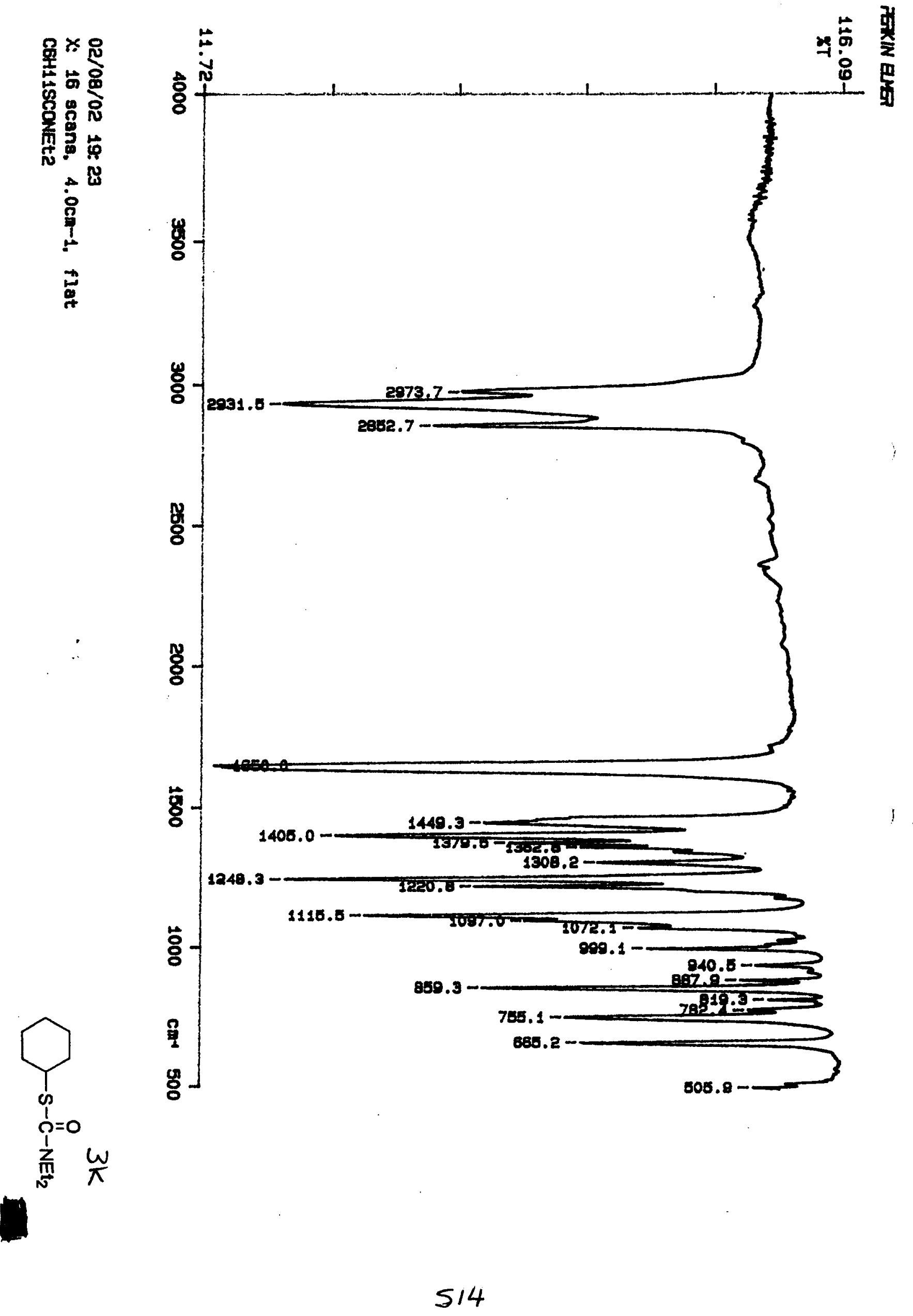




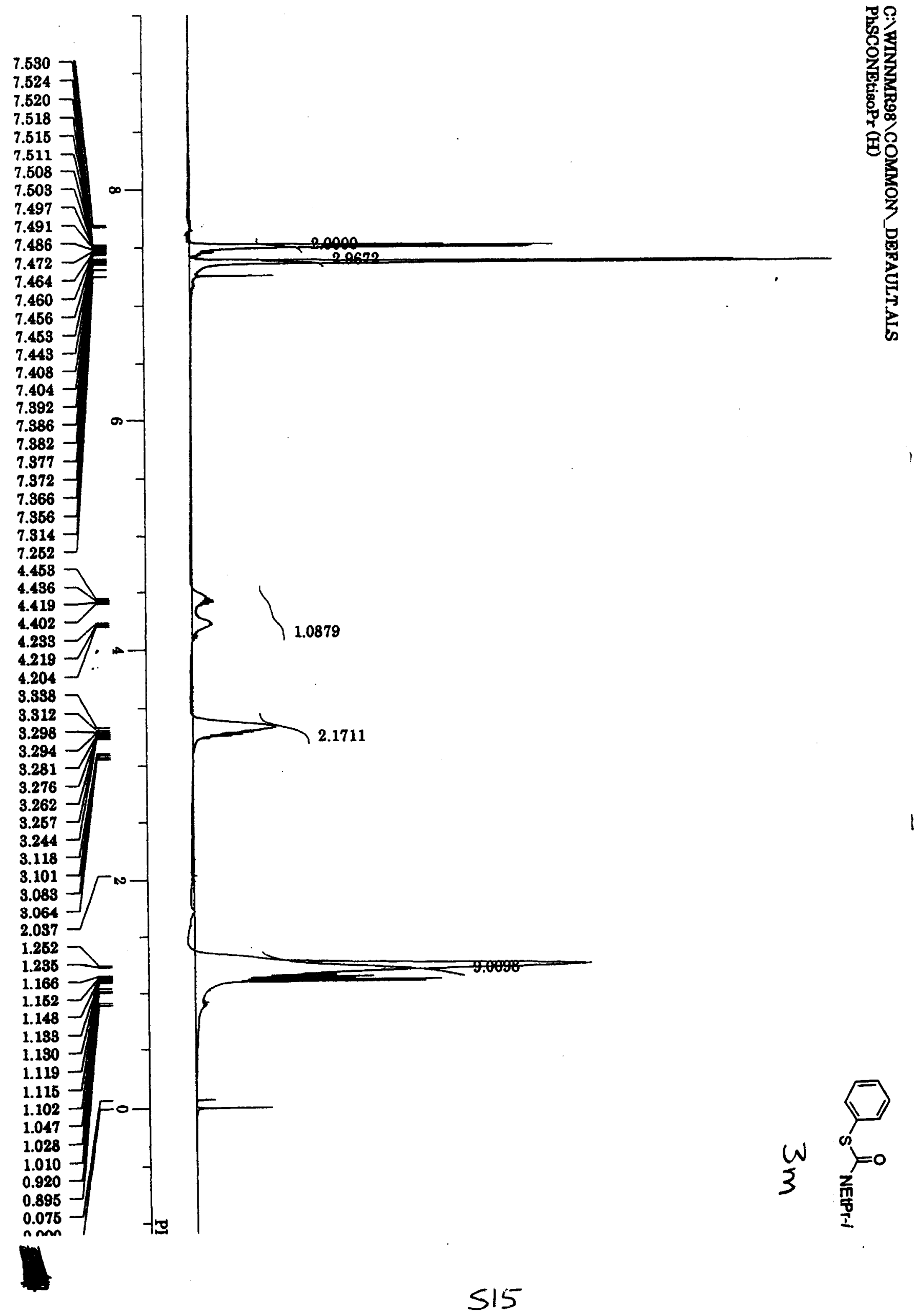




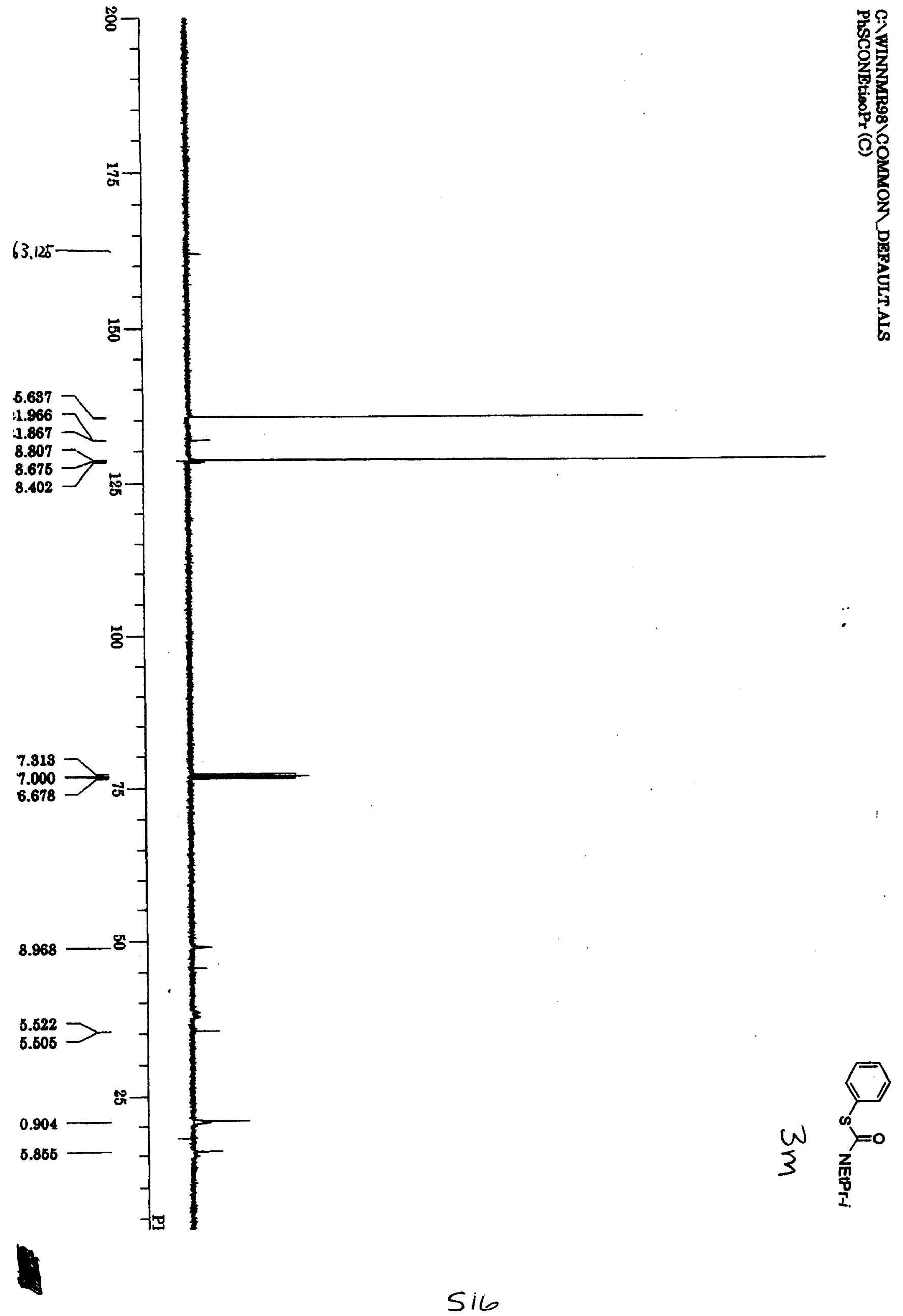




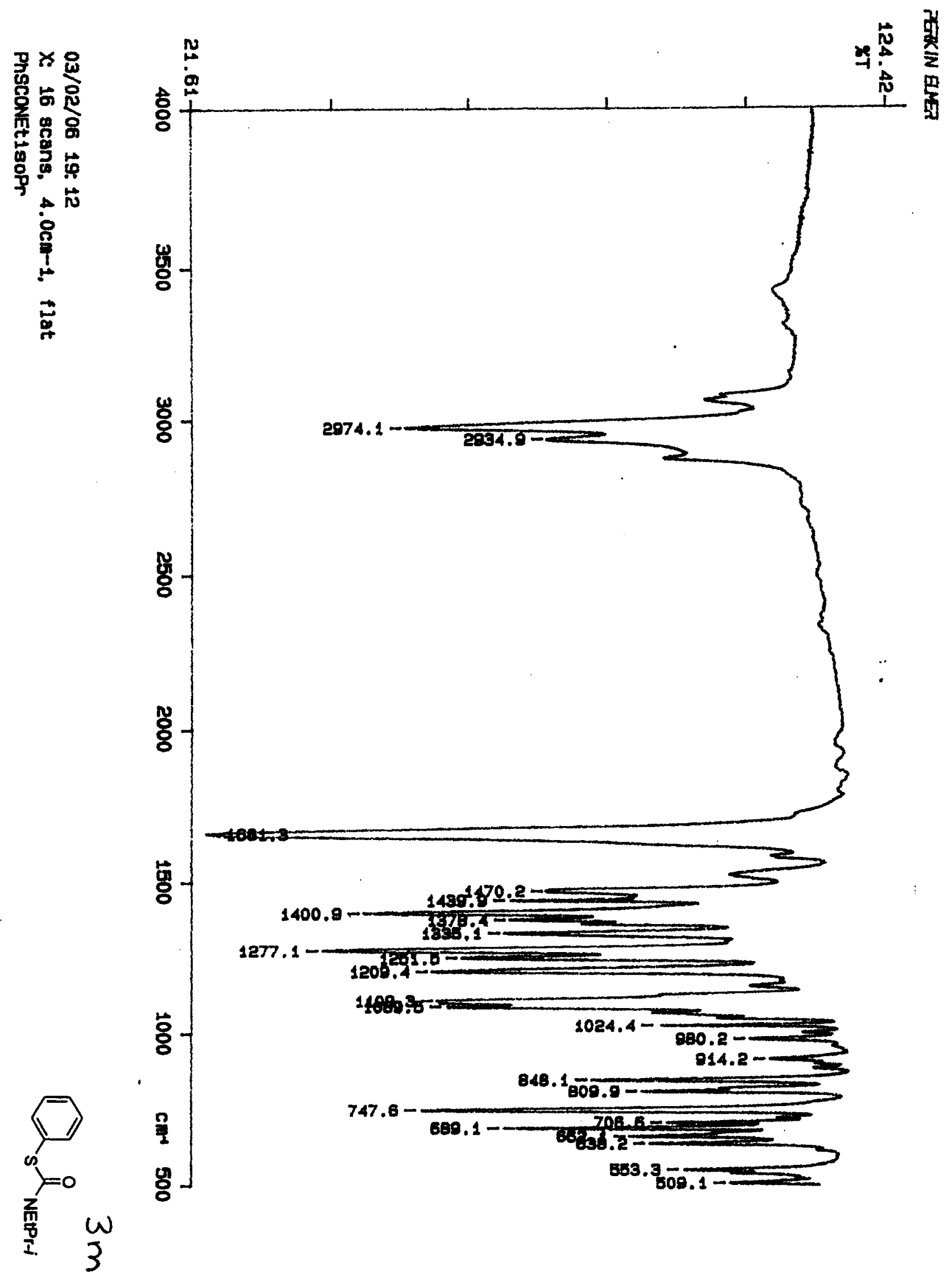

